\title{
Routine Sensor-Augmented Pump Therapy in Type 1 Diabetes: The INTERPRET Study
}

\author{
Kirsten Nørgaard, MD, DMSc, Andrea Scaramuzza, MD, ${ }^{2}$ Natasa Bratina, MD, PhD, ${ }^{3}$ \\ Nebojsa M. Lalić, MD, PhD, ${ }^{4}$ Przemyslaw Jarosz-Chobot, MD, PhD, ${ }^{5}$ Győző Kocsis, MD, \\ Edita Jasinskiene, MD, ${ }^{7}$ Christophe De Block, MD, $\mathrm{PhD}_{1}^{8}$ Odile Carrette, $\mathrm{PhD}^{9}$ \\ Javier Castañeda, MSc, ${ }^{10}$ and Ohad Cohen, MD, ${ }^{11}$ for the INTERPRET Study Group*
}

\begin{abstract}
Background: Sensor-augmented pump (SAP) therapy can improve glycemic control, compared with multiple daily insulin injections or with insulin pump therapy alone, without increasing the risk of hypoglycemia.

Subjects and Methods: A 12-month observational study in patients with type 1 diabetes treated with continuous subcutaneous insulin infusion (CSII), upon the introduction of continuous glucose monitoring (CGM), was conducted in 15 countries (in Europe and in Israel) to document the real-life use of SAP and assess which variables are associated with improvement in type 1 diabetes management.

Results: Data from 263 patients (38\% male; mean age, $28.0 \pm 15.7$ years [range, 1-69 years]; body mass index, $23.3 \pm 4.9 \mathrm{~kg} / \mathrm{m}^{2}$; diabetes duration, $13.9 \pm 10.7$ years; CSII duration, $2.6 \pm 3$ years) were collected. Baseline mean glycated hemoglobin A1c $\left(\mathrm{HbA}_{1 \mathrm{c}}\right)$ was $8.1 \pm 1.4 \%$; $82 \%$ had suboptimal $\mathrm{HbA}_{1 \mathrm{c}}(\geq 7 \%)$. The average sensor use for 12 months was 30\% (range, 0-94\%), and sensor use decreased with time (first 3 months, 37\%; last 3 months, $27 \%$ ). Factors associated with improvement in $\mathrm{HbA}_{1 \mathrm{c}}$ after 12 months in patients with baseline $\mathrm{HbA}_{1 \mathrm{c}} \geq 7 \%$ were high baseline $\mathrm{HbA}_{1 \mathrm{c}}(P<0.001)$, older age group $(P<0.001)$, and more frequent sensor use $(P=0.047)$. Significantly less hospitalization, increased treatment satisfaction, and reduced fear of hypoglycemia were reported after 12 months of SAP.

Conclusions: This is the largest and longest multicenter prospective observational study providing real-life data on SAP. These results are consistent with those of controlled trials showing the effectiveness of CGM in pump users.
\end{abstract}

\section{Introduction}

C ONTINUOUS GLUCOSE MONITORING (CGM) systems represent an important advance in diabetes technology that can facilitate optimal glucose control in type 1 diabetes. Numerous randomized controlled trials have demonstrated the safety and efficacy of real-time CGM in both suboptimally and well-controlled type 1 diabetes patients. ${ }^{1-6}$ In addition, recent studies demonstrated the superiority of sensor- augmented pump (SAP) therapy versus multiple daily injections, ${ }^{7,8}$ with a mean decrease of $0.8-1.2 \%$ in glycated hemoglobin $\mathrm{A} 1 \mathrm{c}\left(\mathrm{HbA}_{1 \mathrm{c}}\right)$ levels, without increasing the risk of hypoglycemia. In all these trials, the benefits of CGM correlate with frequent sensor wear and more advanced age., 90

Evaluation of technological interventions is complicated by a frequent lack of generalizability of randomized clinical trial results to everyday clinical settings. Often, randomized clinical trial results reflect not only the technology tested, but also

\footnotetext{
${ }^{1}$ Hvidovre University Hospital, Hvidovre, Denmark.

${ }^{2}$ Luigi Sacco Hospital, Milan, Italy.

${ }^{3}$ University Medical Center-University Children's Hospital, Ljubljana, Slovenia.

${ }^{4}$ Clinic for Endocrinology, Clinical Center of Serbia, University of Belgrade Faculty of Medicine, Belgrade, Serbia.

${ }^{5}$ Medical University of Silesia, Katowice, Poland.

${ }^{6}$ Péterfy Hospital, Budapest, Hungary.

${ }^{7}$ Institute of Endocrinology, Lithuanian University of Health Sciences, Kaunas, Lithuania.

${ }^{8}$ Department of Endocrinology-Diabetology, Antwerp University Hospital, Antwerp, Belgium.

${ }^{9}$ Medtronic International Trading SàrL, Tolochenaz, Switzerland.

${ }^{10}$ Medtronic Bakken Research Center, Maastricht, The Netherlands.

${ }^{11}$ Chaim Sheba Medical Center, Tel Hashomer, Israel.

*A complete list of the members of the INTERPRET Study Group can be found in the Appendix.

This study is registered at ClinicalTrials.gov with trial registration number NCT00790088.
} 
the somewhat rarefied trial setting itself, involving highly qualified healthcare teams with ample skill, time, and resources to assist patients in implementing the technology and to encourage protocol adherence. In clinical practice, SAP therapy is indicated for patients who cannot achieve good metabolic control on continuous subcutaneous insulin infusion (CSII), who have a history of severe hypoglycemia and/ or hypoglycemia unawareness, or who desire increased flexibility in their daily lives while maintaining or even improving their $\mathrm{HbA}_{1 \mathrm{c}}$ levels. ${ }^{11-13}$

The aim of this multicenter prospective observational study was to document in real-life settings the long-term sensor usage and safety of SAP in a broad cohort of patients with type 1 diabetes, to assess which variables are associated with improvement in $\mathrm{HbA}_{1 \mathrm{c}}$, and to record each patient's selfreported outcomes. Preliminary observations of this study have been presented in abstract form. ${ }^{14,15}$

\section{Subjects and Methods}

The INTERPRET Study was designed as a prospective observational study that included 27 investigational centers in 15 countries (in Europe and in Israel). Physicians treated their enrolled patients under routine care, conducting visits approximately every 3 months over the course of 1 year. Inclusion criteria included a diagnosis of type 1 diabetes mellitus with at least 6 months of CSII treatment prior to enrollment, and the insulin pump had to be compatible with real-time display of CGM data. Only the Paradigm ${ }^{\circledR}$ REAL-Time ${ }^{\mathrm{TM}}$ and Paradigm $\mathrm{Veo}^{\mathrm{TM}}$ pumps (Medtronic MiniMed, Inc., Northridge, $\mathrm{CA}$ ) met this criterion at the time of the study; Sofsensor $^{\mathrm{TM}}$ subcutaneous glucose sensors (Medtronic MiniMed, Inc.) were used exclusively. The usual prescribed blood glucose meter of choice was used. The exclusion criterion was prior use of CGM devices with real-time data display. There were no restrictions on age or baseline $\mathrm{HbA}_{1 \mathrm{c}}$ values. No devices were provided by the study sponsor.

Participating physicians were required to have experience in treating patients with real-time CGM and to recommend that sensors be worn for at least $10 \%$ of the time per year. The decision to initiate SAP therapy was made prior to and independently of the study. The devices were prescribed according to their instructions for use. Pump settings, operations with the CGM, and alarms for hypoglycemia and hyperglycemia were set by the treating healthcare professional according to the patients' individual needs and could be freely adjusted during the course of the study. $\mathrm{HbA}_{1 \mathrm{c}}$ values were centrally determined from samples obtained at baseline and at 3, 6, 9, and 12 months with a Diabetes Control and Complication Trial-aligned high-performance liquid chromatography method (Laboratorium für Klinische Forschung, Schwentinental, Germany). The $\mathrm{HbA}_{1 \mathrm{c}}$ values were available to investigators, staff, and patients. Validated versions of Diabetes Treatment Satisfaction Questionnaires Status and Change versions (DTSQs and DTSQc, respectively) $)^{16,17}$ and Hypoglycemia Fear Survey-II (HFS) questionnaires ${ }^{18}$ were administered to adults in Danish, Hungarian, and Spanish centers only, where validated translated versions of the questionnaires were available at that time. The DTSQc questionnaire was used to assess the perceived relative change in satisfaction compared with the previous treatment that could perhaps not be detected by the DTSQs in the case of a relatively high level of patient satisfaction at baseline (ceiling effect). The questionnaires were handed out at baseline (DTSQs and HFS), 3 months (DTSQs), 6 months (HFS), and 12 months (DTSQs, DTSQc, and HFS).

Management decisions were made as per routine practice by the patients and physician-investigators and were based in part on CGM and $\mathrm{HbA}_{1 \mathrm{c}}$ data. Patients could upload data from their devices using CareLink ${ }^{\mathrm{TM}}$ Personal therapy management software (Medtronic MiniMed, Inc.) for additional data visualization options, as needed.

The study was conducted according to the principles of the Declaration of Helsinki on biomedical research involving human patients and the respective local national regulations. Each center obtained local Ethics Committee approval before beginning patient recruitment. Written informed consent was obtained from all patients before enrollment. For the participants $<18$ years of age, both children (whenever possible) and parents (or legal representative) signed informed consent forms. Baseline data included pump and compatible blood glucose meter data, which were uploaded to CareLink Clinical software (Medtronic MiniMed, Inc.). Safety data including serious adverse events, whether related or unrelated to the study device, were also recorded. Serious adverse events included severe hypoglycemia (defined as an episode absolutely requiring assistance from another person and preferably accompanied by a confirmatory blood glucose value of $<50 \mathrm{mg} / \mathrm{dL}[<2.8 \mathrm{mmol} / \mathrm{L}])$ and diabetic ketoacidosis/severe hyperglycemia (defined as a blood glucose value of $>250 \mathrm{mg} / \mathrm{dL}$ [ $>13.9 \mathrm{mmol} / \mathrm{L}]$ with either low serum bicarbonate $[<15 \mathrm{mEq} / \mathrm{L}]$ or low $\mathrm{pH}[<7.3]$ and either ketonemia or ketonuria and requiring treatment within a healthcare facility).

To calculate the percentage of time that sensors were used, the number of valid sensor readings was expressed as a percentage of the maximum possible number of daily readings (i.e., 288) during the observation period. Patients were categorized in different sensor usage frequency subgroups as 0 to $<10 \%, 10$ to $<25 \%$, 25 to $<50 \%, 50$ to $<75 \%$, and $75-100 \%$. Similarly, age was treated as a categorical variable using different subgroups (Table 1). To assess whether there are factors predictive of changes in $\mathrm{HbA}_{1 \mathrm{c}}$ value from the start of $\mathrm{SAP}$ therapy to the last visit at 12 months, a multiple regression model was used with baseline $\mathrm{HbA}_{1 \mathrm{c}}$ value, sensor usage group during 12 months, age group, the main indication for SAP initiation, the duration of CSII prior to CGM initiation, retraining on sensor use during the study, Bolus Wizard ${ }^{\circledR}$ (Medtronic MiniMed, Inc.) use, and CareLink Personal use. The regression model was fitted for patients with a baseline $\mathrm{HbA}_{1 \mathrm{c}}$ value of $\geq 7 \%$. Patient demographics and baseline characteristics have been presented using appropriate summary statistics. Patient-reported outcomes (DTSQs and HFS) were analyzed with linear mixed models with subject-specific random intercepts and mean structure consisting of the fixed effects: visit, sensor usage group, baseline $\mathrm{HbA}_{1 \mathrm{c}}$ value, and the main indication for SAP initiation. Dunnett's test was used for pairwise comparisons. For analysis of the DTSQc data, where scores were assessed at the 12-month visit, a multiple regression model was used. A negative binomial model with subject-specific random intercepts was used for the analysis of hospitalizations. At the end of the study, patients were asked to complete a user survey to collect their feedback on the SAP treatment. The items were scored from 0 (strongly disagree) to 


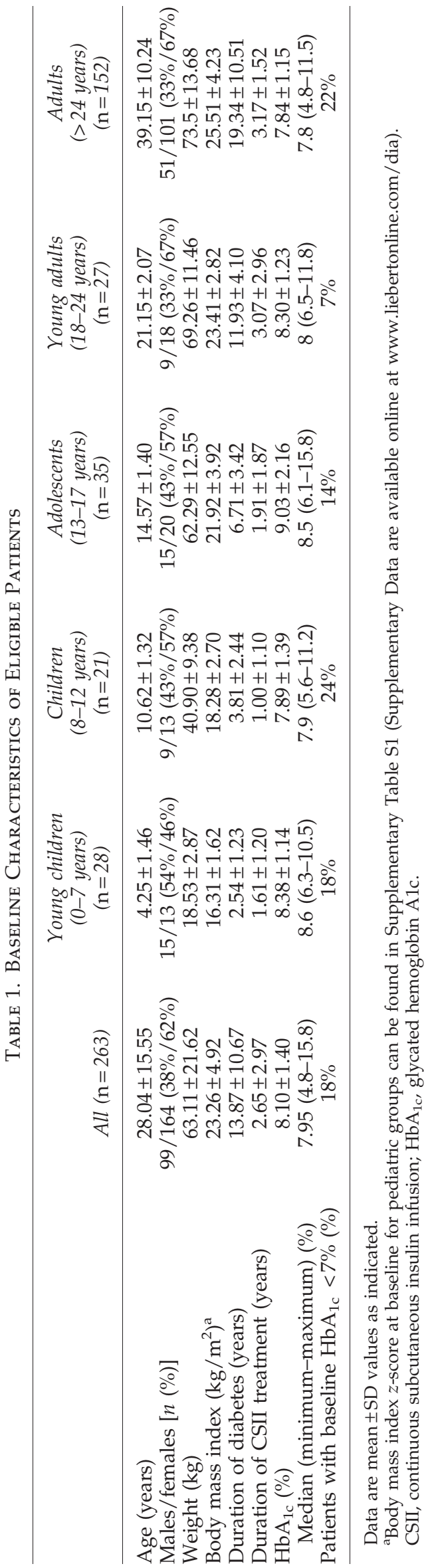

10 (strongly agree). An average score of $\geq 8$ was considered as "strongly agree."

\section{Results}

The 27 active sites in 15 countries recruited a total of 274 patients. At baseline, few patients had to be excluded from analyses because of major protocol deviations such as missing patient informed consent (one patient), CSII therapy for less than 6 months (two patients), prior experience with SAP therapy (seven patients), and no electronic Case Report Form data information available (one patient). Hence 263 patients were eligible for analyses at baseline; among these individuals, 84 were $<18$ years of age. Baseline characteristics of eligible patients are summarized in Table 1 . On average, the patients had $2.65 \pm 2.97$ years of CSII experience (95\% confidence interval $[\mathrm{CI}] 2$ years). Mean $\pm \mathrm{SD} \mathrm{HbA}_{1 \mathrm{c}}$ levels at the time of initiation of CGM were $8.1 \pm 1.4 \%$ (range, $4.8-15.8 \%$ ); 212 $(81.5 \%)$ subjects had a baseline $\mathrm{HbA}_{1 \mathrm{c}}$ value of $\geq 7.0 \%$. The main indications for SAP therapy initiation were glycemic instability (38\%), persistently high $\mathrm{HbA}_{1 \mathrm{c}}(30 \%)$, recurrent hypoglycemia $(8 \%)$, hypoglycemia unawareness $(8 \%)$, and flexibility/lifestyle or patient choice $(10 \%)$. The study was completed by 235 (89\%) of the 263 subjects; four patients stopped CSII therapy during the 12-month study period, seven patients were lost to follow-up despite every attempt from the treating physician to contact them, and 17 enrolled subjects did not continue the study for other reasons (e.g., changed treating physician, left the clinic, lack of time, moved countries).

\section{CGM use}

The average sensor use for the complete observation period was $30 \%$ of the time (range, 0-94\%). Usage decreased over time from $37 \%$ in the first 3 months to $27 \%$ in the last 3 months $(P<0.0001)$ (Fig. 1). Twenty-four percent of the patients used the sensor at least half of the time during the 12-month observation period, and $19 \%$ of the patients used it for $<10 \%$ of the time. At the beginning of the study, $40 \%$ of the patients were advised by their physicians to wear sensors more than $75 \%$ of the time, wearing the sensor was recommended to $18 \%$ for $50-74 \%$ of the time, $8 \%$ for $25-49 \%$ of the time, and $29 \%$ for $10-24 \%$ of the time, and $5 \%$ of the patients received the recommendation to wear the sensor less than $5 \%$ of the time for the first 3 months. These recommendations were sometimes changed during the course of the study. The sensor training was performed during the baseline visit for $98.9 \%$ of the patients and was delivered as one unique session in most of the cases $(81.5 \%)$. During this training, $85.9 \%$ of the participants were trained on the use of the real-time information (trends and alerts) to which CGM gives them access on their pump screen. The characteristics of participants were analyzed to identify potential predictors of long-term high sensor use ( $\geq 75 \%$ of the time). A low baseline $\mathrm{HbA}_{1 \mathrm{c}}$ and high sensor use during the first 3 months were factors significantly associated with high sensor use during the 12-month period $(P=0.015$ and $P=0.001$, respectively). No significant effect of age, gender, duration of diabetes or CSII therapy, main indication for SAP therapy initiation, diabetes-related complications, way of funding the sensor, retraining on sensor, number of selfmonitoring of blood glucose measurements, or CareLink Personal use was evident. The patients participating to the user survey on SAP treatment $(n=190)$ strongly agreed that 


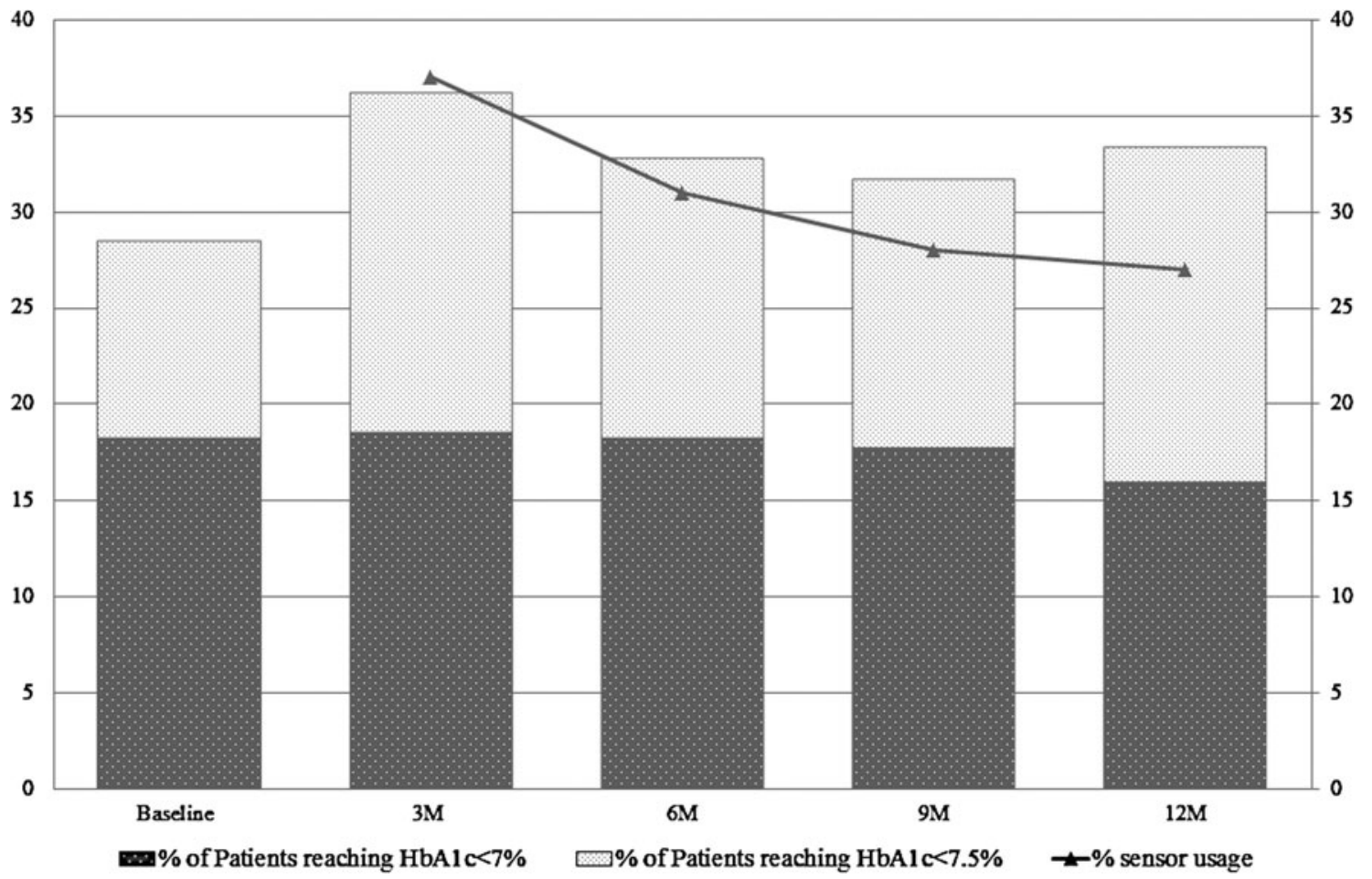

FIG. 1. Sensor use and percentage of the population with glycated hemoglobin A1c (HbA1c) levels of $<7 \%$ and $<7.5 \%$.

the alarms were good at telling them if their glucose was high or low (average score, $8.2 \pm 2.4$ on a 10-point Likert-scale questionnaire) and that the SAP assisted them in achieving better glycemic control (average score, 8.6 \pm 2.0 ).

\section{$\mathrm{Hb}_{1 \mathrm{c}}$ outcome}

The proportion of patients well-controlled with an $\mathrm{HbA}_{1 \mathrm{c}}$ value of $<7 \%$ remained stable throughout the 12 -month observation period (Fig. 1). There were significantly more patients with an $\mathrm{HbA}_{1 \mathrm{c}}$ value of $<7.5 \%$ after 3 months of SAP therapy than at baseline (baseline, 29\%; 3 months, 37\%; Bonferroni corrected $P=0.009$ ). The percentage of patients with an $\mathrm{HbA}_{1 \mathrm{c}}$ value of $<7.5 \%$ decreased with the decrease of sensor usage during the 12-month observation period. The percentage of patients with an $\mathrm{HbA}_{1 \mathrm{c}}$ value of $<7.5 \%$ after 12 months was higher than at baseline, but the difference was not statistically significant (12 months, 33\%; Bonferroni corrected $P=0.185)$. The decrease in $\mathrm{HbA}_{1 \mathrm{c}}$ from baseline to 12 months in patients with a baseline $\mathrm{HbA}_{1 \mathrm{c}}$ value of $\geq 8 \%$ was $0.43 \%$ $(P=0.0014)$. A multiple regression analysis conducted on patients with a baseline $\mathrm{HbA}_{1 \mathrm{c}}$ value of $\geq 7 \%$ showed that factors associated with improvement in $\mathrm{HbA}_{1 \mathrm{c}}$ after 12 months were high baseline $\mathrm{HbA}_{1 \mathrm{c}}$ levels $(P<0.001)$, older age group $(P<0.001)$, and more frequent use of sensor $(P=0.047)$. The main indication for initiation of SAP therapy, the duration of CSII therapy, the retraining on sensor during the study, Bolus Wizard use, and CareLink Personal use were not factors associated with $\mathrm{HbA}_{1 \mathrm{c}}$ decrease after 12 months.

\section{Hospitalization and serious adverse events}

The average number of diabetes-related hospital admissions per 100 patients-years and the average length of stay over the 12-month observation period were significantly reduced compared with the 12 months before starting SAP therapy (25.9 vs. 13.9 per 100 patients-years $[P=0.0002]$ and $1.7 \pm 5.0$ vs. $0.6 \pm 2.6$ days $[P<0.001]$, respectively). There was a decrease in the number of admissions / 100 patients-years in the overall population when sensor use increased, although the difference was not significant $(P=0.0697)$. During the 12 months prior to initiation of SAP therapy, five patients were hospitalized because of diabetic ketoacidosis (DKA), whereas no hospitalizations for severe hypoglycemia were reported. During the observation period, three patients required a total of three hospital admissions for treatment of DKA, and nine patients reported 13 episodes of severe hypoglycemia, of which only four events required emergency room visit or hospitalization. The change in the rate of DKA and severe hypoglycemia was not statistically significant. The overall incidence of severe hypoglycemic events was 6.3 events per 100 patient-years, and the ketoacidosis rate was 1.1 per 100 patient-years. Six other diabetes-related (angioplasty, diabetic foot ulcer, ischemic heart disease, pancreas and liver transplantations, suicide attempt, and poor glycemic control) and six non-diabetes-related serious adverse events that were not related to the study device were reported.

\section{Patient-reported outcomes}

The DTSQs and DTSQc questionnaires were administered to 66 eligible patients; 40 patients returned the entire HFS questionnaire at 12 months, and 20 patients completed only the Worry subscale as the translation of the Behavior subscale was not fully validated in one of the selected languages. Patients reported highly favorable scores for treatment satisfaction on the DTSQs questionnaire at baseline (average score, 32 out of 36 possible points), and this score remained 
unchanged throughout the study period. In addition, patients reported a significant decrease in the perceived frequency of hyperglycemic episodes after 12 months $(P=0.047)$. All patients gave favorable responses to the treatment satisfaction questions at 12 months on the DTSQc questionnaire (average score, $13.6 \pm 5.7$ out of $18 ; 95 \%$ CI $[12.2,14.9])$, indicating improved treatment satisfaction compared with previous treatment. The perceived frequency of hyperglycemia did not change (average score, $-0.4 ; 95 \%$ CI $[-0.8,0.0]$ ), and the perceived frequency of hypoglycemia was reduced (average score, $-0.62 ; 95 \%$ CI $[-1.0,-0.2])$.

The total HFS score was significantly improved versus baseline after 12 months of treatment (Fig. 2). The modelbased average change on the total HFS score at 12 months was $-6.87(95 \%$ CI $[-11.54,-2.20] ; P=0.003)$. Scores in the "behaviors to avoid hypoglycemia" and "worries about hypoglycemia" subscales were significantly improved over the 12 months of treatment with CGM: the model-based average changes were -2.6 and $-4.1(95 \% \mathrm{CI}[-4.87,-0.31], P=0.024$ and $[-6.91,-1.30], P=0.003$, respectively). There was significant improvement in the "worry" subscale between 12 months and baseline (average change -5.21 , 95\% CI [-9.71, $-0.70], P=0.021$ after adjustment for sensor use).

\section{Discussion}

This is the largest and longest observational study of reallife SAP use in Europe and Israel to date. Our study confirms the incremental benefit of sensor use in the population of CSIItreated patients requiring the use of CGM for various indications. Higher baseline $\mathrm{HbA}_{1 \mathrm{c}}$, older age at initiation of SAP therapy, and high sensor use were associated with greater $\mathrm{HbA}_{1 \mathrm{c}}$ improvements. There were no demographic characteristics that were associated with continuous use of sensor (more than $75 \%$ of the time) for a period of 12 months. Only patients with high sensor use at the beginning of SAP as well as patients with lower $\mathrm{HbA}_{1 \mathrm{c}}$ at baseline tended to wear the sensor more often in the 12-month observation period. One can hypothesize that well-controlled patients are likely to seek continuous protection against hypoglycemia or hypoglycemia and hyperglycemia (a strict control). By contrast, patients who do not understand or practice the basics of intensive insulin therapy still have the greatest challenges with efficient adoption of SAP therapy. These results are consistent with recent controlled studies comparing CGM versus selfmonitoring of blood glucose ${ }^{9}$ and SAP versus multiple daily injections. ${ }^{19}$ Ours is the first study to identify continuous use of sensor for 3 months as a factor predictive of high use in the long term, suggesting that patients' willingness to wear the sensor consistently is influenced by the initial favorable experiences with high use. No other parameters have been detected to help physicians at the outset of the therapy in identifying the best candidates for SAP. Further observational studies with large enough and more refined populations could potentially answer this still-open question.

Despite evidence that a clinically relevant benefit can be expected if the patient wears the sensor more than $70 \%$ of the time $^{5,6}$ and clinical guidelines emphasizing the need to use the sensor continuously, ${ }^{11,13}$ this study reports a low sensor use in this population. This is only partly explained from the lack of reimbursement of sensors in most of the countries participating in the study because some hospital and charity organizations are able to fund sensors. One interesting finding of this report is the wide range of sensor usage recommendations given by physicians. Some physicians were potentially unaware of the benefits of CGM technology or are still not compellingly advising the use of sensors according to guidelines and published recommendations. The lack of sensors reimbursement may be a disincentive. Other physicians did not recommend continuous use because of flexibility/lifestyle concerns or skin irritation.

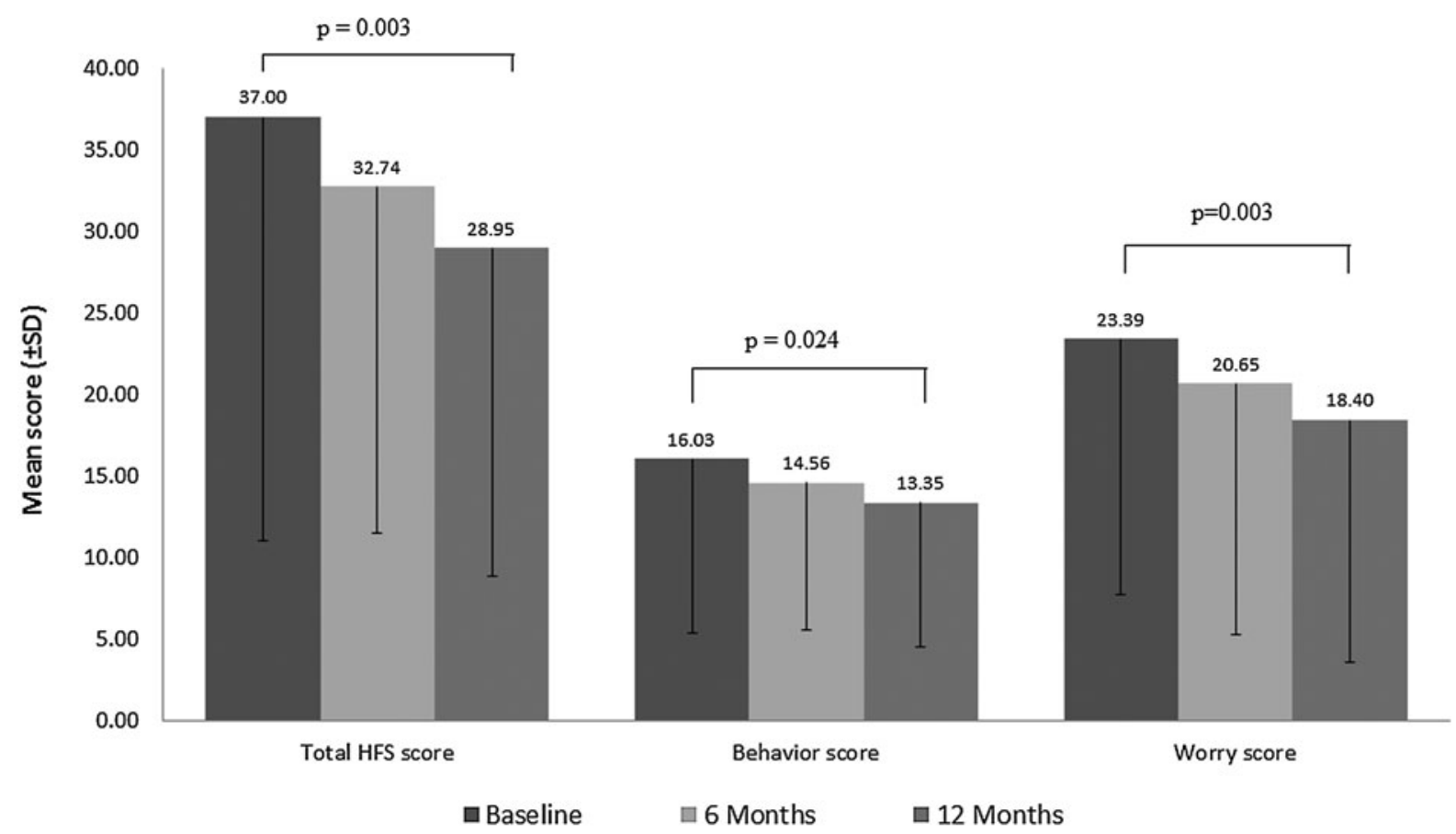

FIG. 2. Hypoglycemia Fear Survey-II (HFS) scores at baseline, 6 months, and 12 months. 
Despite the relatively low sensor use on average, the assessment of patients' perceived fear of hyperglycemia or severe hypoglycemia improved significantly. This study indicates that CGM in real-life settings led to a significant decrease in patients' fear of hypoglycemia and provides additional evidence of the favorable impact of CGM on patients' treatment satisfaction. We were not able to administer the questionnaires in all countries participating to the study because of language validation restriction at the time of study start; hence the generalizability of these findings should be interpreted in the context of the characteristics of these study participants. Nevertheless our findings are in line with previous studies. ${ }^{8,25}$ The rates of severe hypoglycemia and DKA found in our study are relatively low versus rates reported in the literature ${ }^{20}$ but similar to those observed in a study in a large cohort of children evaluated retrospectively in a real-life setting. ${ }^{21}$ The number of severe hypoglycemic episodes that did not lead to hospitalization in the previous 12 months before the study was not collected, although we asked for severe hypoglycemia reporting at each patient visit. Therefore, we cannot draw strong conclusions on the effect of SAP therapy on severe hypoglycemia. We observed that a small number of events happened while the sensor was supposedly protecting the patient from severe hypoglycemia. None of these patients was using a pump equipped with a shut-off system that could have automatically suspended insulin delivery when sensor values reached critical lows. ${ }^{22,23}$ The use of SAP revealed similar results concerning the incidence of DKA, with slightly lower rates of diabetic acidosis reported during the observation period and only one occurring on a day the patient was wearing a sensor. We observed significantly lower hospitalization rates and lengths of stay when comparing the 12-month data collected at baseline (retrospective collection) versus the 12-month observation, suggesting that physicians did not consider it necessary to hospitalize the patients for pump training, glycemic variability assessment, or education and used the sensor for therapy adjustments in outpatient care.

It has previously been reported that regular clinicianpatient contact is time- and labor-intensive and that there are no agreed strategies for incorporating CGM use into routine clinical care. ${ }^{24}$ We believe that the understanding and interpretation of data from CGM and the relevance of therapeutic interventions are all key elements for the effectiveness of SAP. Because of its real-life setting, our study provides important data in this respect as it highlights several factors associated with successful SAP therapy. Because of its observational nature, it did not have a prospective control group, and hence it was not possible to control for potential confounding factors such as study effects or regression to the mean; as a result, the findings related to effectiveness can be considered to be hypothesis-generating only. A before/after approach was applied to several outcomes such as adverse events and rate of hospitalizations. Furthermore, it is noteworthy that the results are consistent with those of controlled trials ${ }^{9,19}$ showing that pump therapy with CGM can achieve effective glycemic control with low risks of hypoglycemia and other complications.

\section{Appendix: The INTERPRET Study Group}

Investigators are listed as (PI) for Principal Investigator, (I) for co-investigator, and (C) for Coordinator: Merete Meld- gaard, RN (C), Hvidovre Hospital, Hvidovre, Denmark; Elisa Giani, MD (I), and Chiara Mameli, MD (I), Ospedale Luigi Sacco, Milan, Italy; Noa Konvalina (C) and Jacob Ilany, MD (I), Chaim Sheba Medical Center, Tel Hashomer, Israel; Tadej Battelino, MD (PI), and Primoz Kotnik, MD (I), Department of Pediatric Endocrinology, University Medical CenterUniversity Children's Hospital, Ljubljana, Slovenia; Aleksandra Jotić, MD (I), and Marija Macesic, MD (I), Clinic for Endocrinology, Clinical Center of Serbia, Belgrade, Serbia; Luc Van Gaal, MD, PhD (I), and Rie Braspenning, RN (C), Department of Diabetology-Endocrinology, Antwerp University Hospital, Antwerp, Belgium; Grazyna Deja, MD, PhD (I), and Mariola Minkina-Pedras, MD, PhD (I), Medical University of Silesia, Katowice, Poland; Gabriella Bádonyi, MD (I), and József Fövényi, MD (I), Péterfy Hospital, Budapest, Hungary; Rasa Verkauskiene, MD (PI), and Ramute Pilkauskiene, RN (C), Endocrinology Clinic, Medical Academy, Lithuanian University of Health Sciences, Kaunas, Lithuania; Eva Örtqvist, MD, PhD (PI), and Anna-Lena Brorsson, RN (C), Astrid Lindgrens Children's Hospital, Stockholm, Sweden; Katarzyna Cyganek, MD, PhD (I), and Tomasz Klupa, MD, PhD (I), Oddzial Kliniczny Kliniki Chorób Metabolicznych Sz, Kraków, Poland; Ellen Grodum, MD (PI), and Ulla L. Jørgensen, RN (C), Fredericia Hospital Medical Department, Fredericia, Denmark; Claudio Tubili, MD (PI), and Alessandra Di Flaviani, MD (I), Ospedale S. Camillo Forlanini, Rome, Italy; Pierre Duvezin-Caubet, MD (PI), and Emmanuelle Bourrinet, MD (I), Hôpital de Dax, Dax, France; Didier Gouet, MD (PI), Centre Hospitalier Saint Louis, La Rochelle, France; José Manuel García López, MD (PI), and Marcos Pazos Couselo, RN (C), Division of Endocrinology, Hospital Clínico Universitario de Santiago de Compostela, Santiago de Compostela, Spain; Denisa Janíčková-Žďárská, MD, PhD (I), Fakultní Nemocnice v Motole, Praha, Czech Republic; Bozena Florys, MD (I), II Klinika Chorób Dzieci Uniwersytecki Dzieciecy Szpital Kliniczny im. L. Zamenhofa w Bialymstoku, Bialystok, Poland; Maria Asunción Martínez-Brocca, MD (PI), Endocrinology and Nutrition Unit, Virgen del Rocio University Hospital, Sevilla, Spain; Chris Vercammen, MD (PI), AZ Imelda, Bonheiden, Belgium; Francisco Morales, MD (PI), Hospital Universitario Infanta Cristina, Badajoz, Spain; Lars Stechemesser, MD (I), Universitätsklinik für Innere Medizin I, LKH Salzburg, Salzburg, Austria; Zoltan Taybani, MD (I), Réthy Pál Hospital, Bekescsaba, Hungary; Miroslav Palko, MD (PI), Topcare S.r.o., Košice, Slovakia; and Emil Martinka, MD (PI), National Institute of Endocrinology, Lubochna, Slovakia.

\section{Acknowledgments}

This study was sponsored by and funded by Medtronic International Trading Sàrl. Editorial assistance in the preparation of this article was provided by Dr. Michael Shaw (MScript Ltd., Hove, United Kingdom). Funding for this assistance was provided by Medtronic. The authors are very grateful to the patients and patient's families for their committed participation in the study.

\section{Author Disclosure Statement}

K.N. participates in advisory boards and as a consultant for Roche and for Medtronic. A.S. receives research support and participates in advisory boards for Medtronic. O. Cohen 
declares advisory services, research fees, and study coordination with Medtronic. P.J.-C. received honoraria from Medtronic, Roche, NovoNordisk, Sanofi-Aventis, and Lilly for delivering lectures and workshops. N.B. receives honoraria from Medtronic and NovoNordisk. O. Carrette is an employee of Medtronic International Trading SàrL. J.C. is an employee of the Medtronic Bakken Research Center. N.M.L., G.C., E.J., and C.D.B. declare no competing financial interests exist. K.N., A.S., and O. Cohen discussed and designed the study protocol and researched data. J.C. was in charge of the statistical analyses. O. Carrette coordinated the study and drafted the manuscript. N.B., N.L., P.J.-C., E.J., C.D.B., and G.K. researched data and contributed to the interpretation of results. All authors contributed to the discussion and interpretation of results and reviewed and approved the final manuscript.

\section{References}

1. Deiss D, Bolinder J, Riveline JP, Battelino T, Bosi E, TubianaRufi N, Kerr D, Phillip M: Improved glycemic control in poorly controlled patients with type 1 diabetes using realtime continuous glucose monitoring. Diabetes Care 2006;29:2730-2732.

2. Juvenile Diabetes Research Foundation Continuous Glucose Monitoring Study Group, Tamborlane WV, Beck RW, Bode BW, Buckingham B, Chase HP, Clemons R, Fiallo-Scharer R, Fox LA, Gilliam LK, Hirsch IB, Huang ES, Kollman C, Kowalski AJ, Laffel L, Lawrence JM, Lee J, Mauras N, O'Grady M, Ruedy KJ, Tansey M, Tsalikian E, Weinzimer S, Wilson DM, Wolpert H, Wysocki T, Xing D: Continuous glucose monitoring and intensive treatment of type 1 diabetes. $\mathrm{N}$ Engl J Med 2008;359:1464-1476.

3. Juvenile Diabetes Research Foundation Continuous Glucose Monitoring Study Group: The effect of continuous glucose monitoring in well-controlled type 1 diabetes. Diabetes Care 2009;32:1378-1383.

4. O'Connell MA, Donath S, O'Neal DN, Colman PG, Ambler GR, Jones TW, Davis EA, Cameron FJ: Glycaemic impact of patient-led use of sensor-guided pump therapy in type 1 diabetes: a randomised controlled trial. Diabetologia 2009; 52:1250-1257.

5. Raccah D, Sulmont V, Reznik Y, Guerci B, Renard E, Hanaire $\mathrm{H}$, Jeandidier $\mathrm{N}$, Nicolino M:Incremental value of continuous glucose monitoring when starting pump therapy in patients with poorly controlled type 1 diabetes. The RealTrend Study. Diabetes Care 2009;32:2245-2250.

6. Battelino T, Conget I, Olsen B, Schütz-Fuhrmann I, Hommel E, Hoogma R, Schierloh U, Sulli N, Bolinder J; SWITCH Study Group: The use and efficacy of continuous glucose monitoring in type 1 diabetes treated with insulin pump therapy: a randomised controlled trial. Diabetologia 2012; 55:3155-3162.

7. Bergenstal RM, Tamborlane WV, Ahmann A, Buse JB, Dailey G, Davis SN, Joyce C, Peoples T, Perkins BA, Welsh JB, Willi SM, Wood MA; STAR 3 Study Group: Effectiveness of sensor-augmented insulin-pump therapy in type 1 diabetes. N Engl J Med 2010;363:311-320.

8. Hermanides J, Nørgaard K, Bruttomesso D, Mathieu C, Frid A, Dayan CM, Diem P, Fermon C, Wentholt IM, Hoekstra JB, DeVries JH: Sensor-augmented pump therapy lowers $\mathrm{HbA}_{1 \mathrm{c}}$ in suboptimally controlled type 1 diabetes; a randomized controlled trial. Diabet Med 2011;28:1158-1167.
9. Juvenile Diabetes Research Foundation Continuous Glucose Monitoring Study Group, Beck RW, Buckingham B, Miller K, Wolpert H, Xing D, Block JM, Chase HP, Hirsch I, Kollman C, Laffel L, Lawrence JM, Milaszewski K, Ruedy KJ, Tamborlane WV: Factors predictive of use and of benefit from continuous glucose monitoring in type 1 diabetes. Diabetes Care 2009;32:1947-1953.

10. Pickup JC, Freeman SC, Sutton AJ: Glycaemic control in type 1 diabetes during real time continuous glucose monitoring compared with self-monitoring of blood glucose: metaanalysis of randomised controlled trials using individual patient data. BMJ 2011;343:d3805.doi: 10.1136/bmj.d3805.

11. Klonoff DC, Buckingham B, Christiansen JS, Montori VM, Tamborlane WV, Vigersky RA, Wolpert H; Endocrine Society: Continuous glucose monitoring: an Endocrine Society clinical practice guideline. J Clin Endocrinol Metab 2011;96:2968-2979.

12. Blevins TC, Bode BW, Garg SK, Grunberger G, Hirsch IB, Jovanovič L, Nardacci E, Orzeck EA, Roberts VL, Tamborlane WV; AACE Continuous Glucose Monitoring Task Force, Rothermel C: Statement by the American Association of Clinical Endocrinologists Consensus Panel on Continuous Glucose Monitoring. Endocr Pract 2010;16:730-745.

13. Phillip M, Danne T, Shalitin S, Buckingham B, Laffel L, Tamborlane W, Battelino T; Consensus Forum Participants: Use of continuous glucose monitoring in children and adolescents. Pediatr Diabetes 2012;13:215-228.

14. Nørgaard K, Scaramuzza A, Bratina N, Lalić N, JaroszChobot P, Kocsis G, Verkauskiene R, De Block C, Carrette $\mathrm{O}$, Castañeda J, Cohen O; the INTERPRET Study Group: Sensor-augmented pump therapy in real-life: results of the INTERPRET observational study [A-417-000300183]. Oral presentation at the Advances for Technologies and Treatment of Diabetes (ATTD) 2012 Congress, February 2012, Barcelona, Spain.

15. Nørgaard K, Scaramuzza A, Bratina N, Lalić N, JaroszChobot P, Kocsis G, Verkauskiene R, De Block C, Carrette O, Castañeda J, Cohen O; the INTERPRET Study Group: SAP therapy in real life: patient-reported outcomes results of the INTERPRET observational study [abstract 1058]. Diabetologia 2012;55(Suppl 1):pS434.

16. Bradley C: The Diabetes Treatment Satisfaction Questionnaire: DTSQ. In: Bradley C, ed. Handbook of Psychology and Diabetes. A Guide to Psychological Measurement in Diabetes Research and Practice. Chur, Switzerland: Harwood Academic Publishers, 1994.

17. Bradley C: The Diabetes Treatment Satisfaction Questionnaire (DTSQ): change version for use alongside status version provides appropriate solution where ceiling effects occur. Diabetes Care 1999;22:530-532.

18. Gonder-Frederick LA, Schmidt KM, Vajda KA, Greear ML, Singh H, Shepard JA, Cox DJ: Psychometric properties of the Hypoglycemia Fear Survey-II for adults with type 1 diabetes. Diabetes Care 2011;34:801-806.

19. Buse JB, Dailey G, Ahmann AA, Bergenstal RM, Green JB, Peoples T, Tanenberg RJ, Yang Q: Baseline predictors of A1c reduction in adults using sensor-augmented pump therapy or multiple daily injection therapy: the STAR 3 experience. Diabetes Technol Ther 2011;13:601-606.

20. The effect of intensive treatment of diabetes on the development and progression of long-term complications in insulin-dependent diabetes mellitus. The Diabetes Control and Complications Trial Research Group. N Engl J Med 1993;329:977-986. 
21. Scaramuzza AE, Iafusco D, Rabbone I, Bonfanti R, Lombardo L, Schiaffini R, Buono P, Toni S, Cherubini V, Zuccotti GV; Diabetes Study Group of the Italian Society of Paediatric Endocrinology and Diabetology (ISPED): Use of integrated real-time continuous glucose monitoring/insulin pump system in children and adolescents with type 1 diabetes: a 3year follow-up study. Diabetes Technol Ther 2011;13:99-103.

22. Danne T, Kordonouri O, Holder M, Haberland H, Golembowski S, Remus K, Bläsig S, Wadien T, Zierow S, Hartmann $\mathrm{R}$, Thomas A: Prevention of hypoglycemia by using low glucose suspend function in sensor-augmented pump pherapy. Diabetes Technol Ther 2011;13:1129-1134.

23. Garg S, Brazg RL, Bailey TS: Reduction in duration of hypoglycemia by automatic suspension of insulin delivery: the InClinic ASPIRE Study. Diabetes Technol Ther 2012;14:205-209.
24. Joubert M, Reznik Y: Personal continuous glucose monitoring (CGM) in diabetes management: review of the literature and implementation for practical use. Diabetes Res Clin Pract 2011;96:294-305.

25. Juvenile Diabetes Research Foundation Continuous Glucose Monitoring Study Group: Quality-of-life measures in children and adults with type 1 diabetes. Diabetes Care 2010; 33:2175-2177.

Address correspondence to: Kirsten Nørgaard, MD, DMSc Hoidovre University Hospital DK-2650, Hvidovre, Denmark

E-mail: Kirsten.noergaard@regionh.dk 